Nonlinear Processes in Geophysics, 12, 717-723, 2005

SRef-ID: $1607-7946 / \mathrm{npg} / 2005-12-717$

European Geosciences Union

(c) 2005 Author(s). This work is licensed

under a Creative Commons License.

\title{
Dynamics of the burning model
}

\author{
M. Gedalin ${ }^{1}$, M. Bregman ${ }^{1}$, M. Balikhin ${ }^{2}$, D. Coca $^{2}$, G. Consolini ${ }^{3}$, and R. A. Treumann ${ }^{4}$ \\ ${ }^{1}$ Ben-Gurion University, Beer-Sheva, Israel \\ ${ }^{2}$ ACSE, University of Sheffield, Sheffield, UK \\ ${ }^{3}$ Ist. Fisica Spazio Interplanetario, Istituto Nazionale di Astrofisica, Rome, Italy \\ ${ }^{4}$ Max-Planck-Institute for Extraterrestrial Physics, Garching, Germany
}

Received: 4 February 2005 - Revised: 29 March 2005 - Accepted: 1 April 2005 - Published: 19 July 2005

Part of Special Issue "Nonlinear and multiscale phenomena in space plasmas"

\begin{abstract}
We propose a new avalanching model which is characterized by a) a local threshold in the transition from passive to active states, b) finite life time of active sites, and c) is dissipative. This model seems to be more appropriate for the description of a continuous system where localized reconnection plays a crucial role. The model allows for an analytical treatment. We establish the shape of the distribution of cluster sizes and the relation of the observables to the model parameters. The results are illustrated with numerical simulations which support the analytical results.
\end{abstract}

\section{Introduction}

Self-organized criticality (SOC) is a relatively new paradigm (Bak et al., 1987, 1988) that is often expected to explain the statistical behavior of open dynamical systems, such as power law spectra of various variables (Bak et al., 1987). The basic features of such systems are a) random input (driving), b) two regimes (active and passive) of local activity, c) threshold-like transition from the passive to active state, and d) excitation of neighboring sites by the active ones causing the avalanche-like evolution of activity in the system (Jensen, 1998). Such activity may be completely different from the physical point of view. Avalanches can be observed in many systems, like sandpiles (Hwa and Kadar, 1992), earthquakes (Bak and Tan, 1989), forest fires, magnetospheric activity (Chapman et al., 1998; Chang, 1999; Takalo et al., 1999; Consolini and De Michelis, 2001; Valdivia, 2003), solar flares (Lu and Hamilton, 1991; Boffetta et al., 1999; Hamon et al., 2002; Krasnoselskikh et al., 2002), biological evolution (de Boer et al., 1994) etc. Running sandpile models are most ubiquitous, being based on the slope-controlled redistribution rules and immediate passive $\leftrightarrow$ active transitions. In running sandpiles driving (albeit usually a weak one) per-

Correspondence to: $\mathrm{M}$. Gedalin

(gedalin@bgu.ac.il) sists during avalanche development too (Hwa and Kadar, 1992; Corral and Paczuski, 1999). Strictly speaking, SOC occurs only in the limit of infinitely weak driving. In other words, a complete separation of time scales is necessary: the duration of the largest avalanche in the systems should be less than the smallest time interval between subsequent inputs (). This condition is hardly realized in nature, therefore we will not stick with SOC but will discuss avalanching systems in general. This concept is more extended since the behavior of an avalanching system may appear non-SOC, while the basic feature - ability to develop avalanches - remains. It is worth mentioning that it is well-accepted to refer to many avalanching systems as to SOC systems, even not in the limit of zero driving, if these systems exhibit power-law distributions. The latter feature means that the system behavior is scale-free (apart from the smallest possible scale and the size of the system as a whole, as it appears in numerical models). We, nevertheless, will refer to such systems as avalanching, keeping in mind that in real physical systems driving depends on external conditions and may change with time to a become a strong one.

SOC, and sandpile models, in particular, have been widely applied to plasma systems, especially to those which are thought to be governed by localized reconnection (Chang, 1999; Chapman et al., 1998; Charbonneau et al., 2001; Boffetta et al., 1999; Consolini and De Michelis, 2001; Klimas et al., 2004; Krasnoselskikh et al., 2002; Lu and Hamilton, 1991; Takalo et al., 1999; Valdivia, 2003; Uritsky, V., M. Pudovkin, and A. Steen, 2001; Uritsky et al., 2002). The more sophisticated field reversal model (Takalo et al., 1999; Klimas et al., 2004) is based on the hysteresis behavior of the resistivity (diffusion). It is unclear whether it can be directly applied to collisionless localized reconnection. It should be mentioned, though, that there is no definitive observational evidence relating SOC models to the localized reconnection processes in plasma sheet, and there is no general agreement regarding the dynamical nature of current sheet reconnection. Multiple reconnection X lines were evidenced, for example, 
(Slavin et al., 2003), while others reported a single, localized and transient reconnection with an impulsive modulation of reconnection rate (Sergeev et al., 1987; Semenov et al., 1992).

In the present paper we propose and study a new model, which has a number of features characteristic of the localized reconnection process in the current sheet (Milovanov et al., 2001; Milovanov and Zelenyi, 2002; Zelenyi et al., 2002), and which are not, in our opinion, properly presented in other models. These features are:

- the transition passive-to-active depends on the local threshold, resembling what happens in a current layer, when its width becomes less than some critical value, or, alternatively, when the current exceeds the critical current;

- there is a finite life time of an active site, that is, an active site, which excites its neighbour, does not become passive immediately but remains active for some time;

- a part of the energy dissipates into "radiation" which can be observed by a remote observer.

Locality of the threshold can be introduced artificially even in a simple sandpile model by assigning the property of variables to slopes. This works, however, only for onedimensional directed models (otherwise a local vector field of "slopes" has to be defined). Dissipative models are rather ubiquitous (see, e.g. 1995). We, however, propose that the dissipated energy is the energy which comes to a distant observer directly from the active site, and can be used for identification of the system state. The finite life time feature is the most important since it removes the unphysical condition of immediate energy release by an active site with the transition to the passive regime. The proposed feature not only allows to study the behavior of the avalanching system in the fast driving regime, but also makes possible resolution of the temporal behavior of active regions.

In the present paper we study the avalanche properties of the burning model depending on the strength of driving. The question of whether a distant observer can distinguish between the burning system and a sandpile system using only a limited set of measurements is analyzed in the companion paper.

\section{The model}

In this paper we restrict ourselves to a one-dimensional model only (two-dimensional model will be studied elsewhere). Let there be a an array of cells (sites) with the length $L$. Each site $i, i=1, \ldots, L$, is characterized by its temperature $T_{i}$. The system is open and there is an external random heat input. At each time step each site gets the amount of heat $q$ with the probability $p$. Thus, the average heat input into a single site is $q p$ and the total time average driving into the whole system is $q p L$. If the temperature of a site exceeds some critical value, $T_{i} \geq T_{c}$, burning starts, during which the site loses energy at the rate (energy per time step) $J_{i}=k T$. In other words, should this site be left alone, its temperature would change according $\dot{T}=-k T$, or $T=T(0) \exp (-k t)$. Once the temperature drops below another critical value, $T_{i}<T_{0}=s T_{c}, s<1$, the burning ceases, and does not start again until $T_{i} \geq T_{c}$. Physically it corresponds to the idea that the energy release at the reconnecting site, one started, persists until it is exhausted, and can start again only after sufficient energy is accumulated. Summarizing all this, the energy flux is

$J_{i}=k T_{i}\left[\theta\left(T_{i}-T_{c}\right)+\theta\left(T_{c}-T_{i}\right) \theta\left(T_{i}-T_{0}\right) \theta\left(-\dot{T}_{i}\right)\right]$,

where the step-function $\theta(x)=1$ when $x \geq 0$ and zero otherwise. The last term $\theta(-\dot{T})$ in Eq. 1 introduces the history (hysteresis) dependence: if the temperature is below the upper critical value but above the lower critical value, burning occurs only if the site was burning at the previous step, that is, its temperature was decreasing. Note that the description is imprecise since the random input may occasionally cause some reheating even during the burning stage. This problem is easily avoided during the discretization, as we shall see a little later. It is worth mentioning that the life time of a lonely burning site, $t_{l}$, can be estimated as $t_{l} \approx \ln \left(T_{c} / T_{0}\right) / k$.

The energy release $J_{i}$ is partly dissipated, while the rest is isotropically distributed among the neighbors. Let $a<1$ denote the part of the energy which remains in the system. If the temperature of the site $i$ at the time $t$ was $T_{i}(t)$, at the next step it would be

$T_{i}(t+1)=T_{i}(t)-J_{i}(t)+(a / 2)\left(J_{i-1}(t)+J_{i+1}(t)\right)+\eta(i, t)$.

The last term is the random input with the average $\langle\eta\rangle=p q$. Now it is easy to see that the flux can be properly rewritten as

$J_{i}(t)=k T_{i}(t)$

$\left[\theta\left(T_{i}(t)-T_{c}\right)+\theta\left(T_{c}-T_{i}(t)\right) \theta\left(T_{i}(t)-T_{0}\right) \theta\left(J_{i}(t-1)\right)\right]$,

The two Eqs. 2 and 3 completely determine the model. We only have to add the boundary conditions which will be open boundaries, or $T(1)=T(L)=0$ always, and the distribution of the random variable $\eta$. The last one will be usually taken as uniform if not specified otherwise.

The proposed model has the following features which are usually absent (or incomplete) in other models: a) the activepassive transitions depend only on the local conditions, that is, the temperature of the site and the energy release of the same site determine whether it is active or passive, b) a site which becomes active does not fade away immediately once it transfers energy to neighbors, but lives for some time, and c) there is some dissipation along with the energy flow inside the system. These features make the system resembling the a current sheet with localized reconnection going on at different sites. 


\section{Field presentation}

While discretization is the natural and only way for performing numerical simulations, in reality there is nothing to break a continuous system into a number of discrete sites, although some minimal scale is always present in physical systems, like, for example, regular MHD cannot be extended to scales smaller than the ion gyroradius. Thus, a proper analytical description of continuous physical systems would require its formulation in terms of differential equations (in case of history dependence there is no translational symmetry with respect to time and an integro-differentical equation might be expected, in general).

In this section we transform the model into a field model. In order to do this we introduce the respective temporal and spatial reference lengths, $\tau$ and $l$. Thus, $t+1 \rightarrow t+\tau$, and $i \pm 1 \rightarrow x \pm l$. Now Eq. 2 is rewritten as

$\tau \frac{\partial T(x)}{\partial t}=-(1-a) J(x)+\frac{a l^{2}}{2} \frac{\partial^{2} J}{\partial x^{2}}+\eta(x, t)$.

Respectively, the equation for the flux is

$J(t)=k T\left[\theta\left(T(t)-T_{c}\right)+\theta\left(T_{c}-T(t)\right)\right.$

$\left.\theta\left(T(t)-T_{0}\right) \theta(J(t-\tau))\right]$.

The last term in 5 is non-local. Taking into account that all variables change continuously for $T_{0}<T<T_{c}$, one can Taylor expand to get

$\tau \dot{J}=-J+k(T+\tau \dot{T})\left[\theta\left(T+\tau \dot{T}-T_{c}\right)+\right.$

$\theta\left(T_{c}-T-\tau \dot{T}\right) \theta\left(T+\tau \dot{T}-T_{0}\right) \theta(J)$

or

$J=k T\left[\theta\left(T-T_{c}\right)+\theta\left(T_{c}-T\right) \theta\left(T-T_{0}\right) \theta(J-\tau \dot{J})\right]$.

Since the discontinuous $\theta$-functions can be substituted with tanh:

$\theta(x)=\frac{1}{2}\left[1+\tanh \left(\frac{x}{L}\right)^{2 n+1}\right]$

where $L$ is sufficiently small and the integer $n \geq 0$ is sufficiently large, the derived field equations can be written in an explicitly smooth way.

\section{Analytical treatment}

It is easy to estimate the average $N_{a}$ number of active sites, at least for the case of weak to moderate driving. The energy release from each site $\approx T_{c}-T_{0}$ occurs within the time $t_{l}$, so that the average power is $P_{a v} \approx\left(T_{c}-T_{0}\right) / t_{l}$. The total energy loss per unit time (in time step) is $d E / d t \approx-(1-a) N_{a v}\left(T_{c}-T_{0}\right) / t_{l}+(d E / d t)_{b}$. The last term describes the energy loss at the boundaries and does not exceed $-2 k T_{c} \sim T_{c} / t_{l}$. Thus, for a sufficiently large system, $N_{a v}(1-a) \gtrsim 1$ the boundary losses can be neglected in all cases. In the stationary state energy losses should be balanced by the input, which is $L p q$, so that one has

$N_{a v} \approx \frac{L p q t_{l}}{(1-a)\left(T_{c}-T_{0}\right)}$.

This approximation should be valid for $N_{a v} \ll L$, so that

$p q t_{l} \ll(1-a) T_{c}$.

Stronger predictions can be made when using the kinetic equations for the cluster distribution (Gedalin et al., 2005). ${ }^{1}$ The corresponding equation (simplified) for the number of clusters, $N(w)$, with the length $w$ reads:

$$
\begin{aligned}
& \frac{d N(w)}{d t}=-[P(w \rightarrow w+\Delta)+P(w \rightarrow w-\Delta)] N(w) \\
& +P(w-\Delta \rightarrow w) N(w-\Delta)+P(w+\Delta \rightarrow w) N(w+\Delta),
\end{aligned}
$$

where $P(w \rightarrow w+\Delta)$ and $P(w \rightarrow w-\Delta)$ are the probabilities (per unit time) of growth and shrinking, and $\Delta$ is the typical change of length in one step. In our case $\Delta=1$ or $\Delta=2$. Since we are interested in estimates only and will not solve the (discretized) kinetic equation 10 exactly, we simply put $\Delta \approx 1$. We proceed by Taylor expanding to obtain

$\frac{d N}{d t}=\frac{\Delta^{2}}{2} \frac{d^{2}}{d w^{2}}\left(\left(P_{++} P_{-}\right) N\right)-\Delta \frac{d}{d w}\left(\left(P_{+}-P_{-}\right) N\right)$,

where $P_{+}$and $P_{-}$are the growth and shrinking probabilities, respectively. In the stationary state, $(d N / d t)=0$, one has

$\frac{\Delta}{2} \frac{d}{d w}\left(\left(P_{++} P_{-}\right) N\right)=\left(\left(P_{+-} P_{-}\right) N\right)+C$,

where $C=$ const is the probability of the spontaneous appearance of a cluster. Since only clusters of size one are born from the passive background, we have to put $C=0$. Then

$N(w)=\frac{A}{\alpha} \exp \left(-\int \beta d w\right)$,

where $A=$ const, $\alpha=P_{++} P_{-}$, and $\beta=\left(P_{--} P_{+}\right) / \alpha$.

Let us now estimate the growth and shrinking probability. Let the average temperature be $T_{p}$. Since we assume that $N_{a v} \ll L$, this is effectively the average temperature of a passive site. The heat flux from the cluster boundary to the neighboring passive site is $J=k T$ during about its life time. The passive site becomes active if the heat flux exceeds the difference between the critical temperature and the site temperature, which can happen during the time $t_{g}$ when $J>\left(T_{c}-T_{p}\right)$. Estimating the initial temperature of the active site as $\approx T_{c}$ we have $t_{g}=(1 / k) \ln \left[k T_{c} /\left(T_{c}-T_{p}\right)\right]$. The growth probability then will be $P_{+} \approx t_{g} / t_{l}$ and does not depend on the cluster size.

The shrinking probability is simply the probability to find the active site at the boundary in the end of its life, so that $P_{-} \sim 1 / t_{l}$. It also does not depend on the

\footnotetext{
${ }^{1}$ Gedalin, M., Balikhin, B., Coca, D., Consolini, G., and Treumann, R. A.: Kinetic descritption for avalaching systems, http://arxiv.org/pdf/cond-mat/0501567,2005
} 

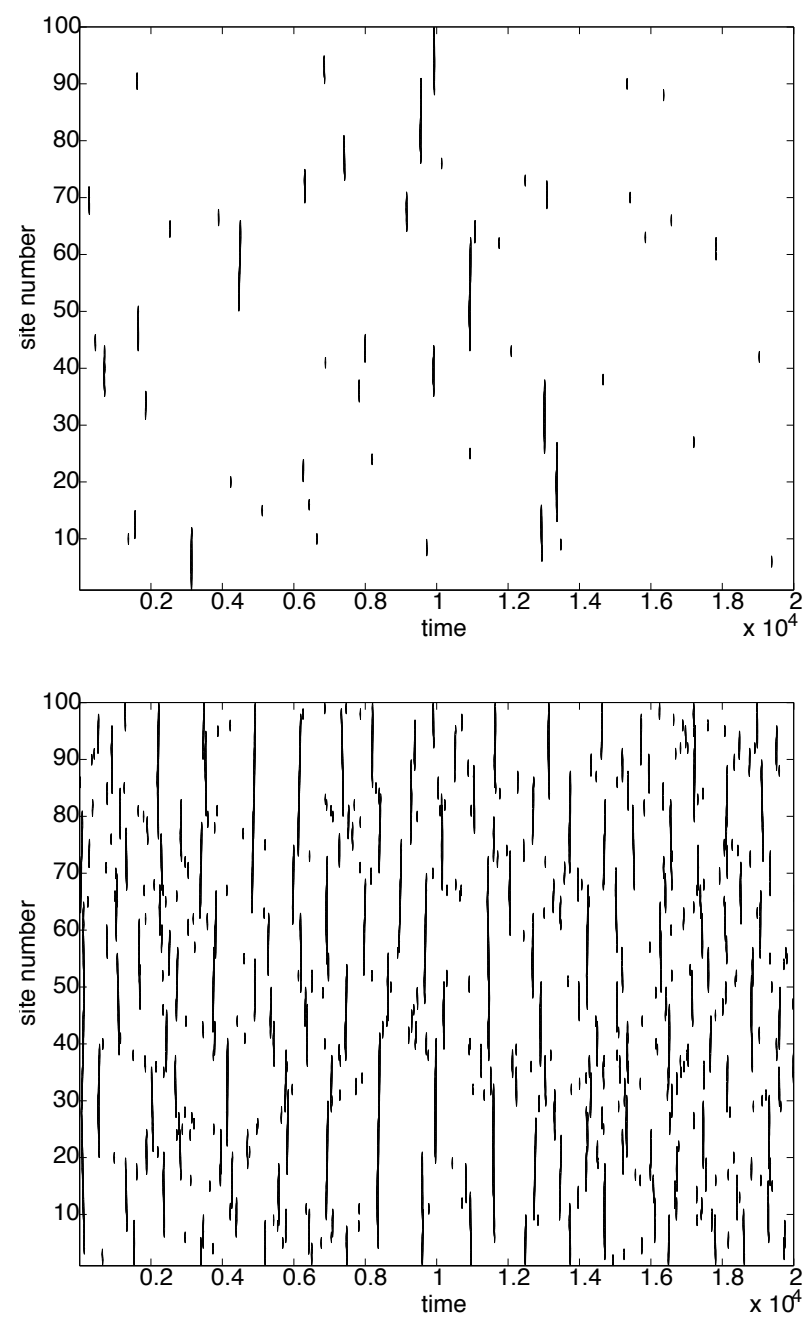

Fig. 1. Energy release for various drivings: top $p=0.0005$ and bottom $p=0.005$.

cluster size. The condition of stationarity immediately gives $T_{p} / T_{c}>1-k \exp (k)$. Respectively, $\alpha=\left(1+t_{g}\right) / t_{l}$ and $\beta=\left(1-t_{g}\right) /\left(1+t_{g}\right)$, so that

$N(w) \propto \exp \left[-\left(1-t_{g}\right) w /\left(1+t_{g}\right)\right] \rightarrow \ln N \propto-w$.

This result appears dependent on the average temperature $T_{p}$ which itself should depend on the system dynamics.

In order to establish the relation of $T_{p}$ to the system parameters we should consider the smallest size, $w=1$, clusters. For such clusters 11 is not applicable. Instead, we have

$\frac{d N_{1}}{d t}=-P_{-} N_{1}+\gamma N_{0}$,

where the last term describes the spontaneous (driving determined) conversion of passive sites into active ones. Since the average driving heat flux into a passive site is $q p$, and the gap $T_{c}-T_{p}$ should be exceeded to make the site active, we can estimate the birth probability $\gamma \sim q p /\left(T_{c}-T_{p}\right)$. In the stationary state we would then have

$\frac{q p N_{0}}{T_{c}-T_{p}}=\frac{N_{1}}{t_{l}}$.

Since $N(w) \propto \exp (-\beta w)$, we have

$N_{a v}=\int_{1}^{L} N(w) d w \approx N_{1} / \beta$,

Substituting 17 into 16, with the use of 8 and taking into account that $N_{0} \approx L$, we get eventually

$\frac{T_{c}-T_{p}}{T_{c}-T_{0}}=\frac{\beta}{1-a}$.

Since $\beta$ itself depends on $T_{p}$, the relation 18 is in fact a (nonlinear) bootstrap equation for the average temperature.

As a by-note, the above analysis allows to predict the maximum size of the cluster. Indeed,

$$
\begin{aligned}
& N(w)=N(1) \exp [-\beta(w-1)] \approx \frac{N_{a v}}{\beta} \exp [-\beta(w-1)] \\
& \approx \frac{L p q t_{l}}{\beta(1-a)\left(T_{c}-T_{0}\right)} \exp [-\beta(w-1)],
\end{aligned}
$$

and one has $N\left(w_{\max }\right)=1$, so that

$w_{\text {max }} \approx \frac{1}{\beta} \ln \frac{L p q t_{l}}{\beta(1-a)\left(T_{c}-T_{0}\right)}$.

Of course, this relation (as all previous) is of approximate character only. It is clear, however, that the average number of active sites depends on the driving more strongly than the maximum cluster size.

\section{Numerical analysis}

The proposed model allows for a pretty good analytical treatment, which nevertheless is based on a number of approximations. Numerical analysis of avalanching models is a usual tool and we expect it to be useful here, at least at the illustrative level.

We start with illustrating the avalanching process by presenting avalanche patterns for various drivings. We use the following parameters: length of the system $L=100$, critical temperature $T_{c}=50$, low critical temperature $T_{0}=0.3 T_{c}$, fraction of energy release going to neighbors $a=0.9$, inverse relaxation time $k=0.3$, and heat amount input at each step $q=2$. The driving strength is then determined by the probability of the input $p$.

Figure 1 shows how the energy release pattern changes with the driving increase by a factor of ten.

Although the difference in the activity level is quite clearly seen visually from Fig. 1, it is instructive to introduce quantitative tools of comparisons. Let $J(t, i)$ be the two-dimensional array of intensities. Then $J_{a v}=\sum_{i, t} J(t, i) /\left(L N_{t}\right)$, where $N_{t}$ is the number of time steps, would have the meaning of the mean site intensity. 
Table 1. Quantitative comparison of activity level.

\begin{tabular}{lllll}
\hline & $p=0.0005$ & $p=0.001$ & $p=0.005$ & $p=0.01$ \\
\hline$q p$ & 0.001 & 0.002 & 0.01 & 0.02 \\
$J_{a v}$ & 0.011 & 0.026 & 0.12 & 0.24 \\
$n_{a v}$ & 0.0013 & 0.0032 & 0.0145 & 0.029 \\
$w_{\max }$ & 15 & 18 & 21 & 22 \\
$t_{\max }$ & 32 & 37 & 37 & 36
\end{tabular}
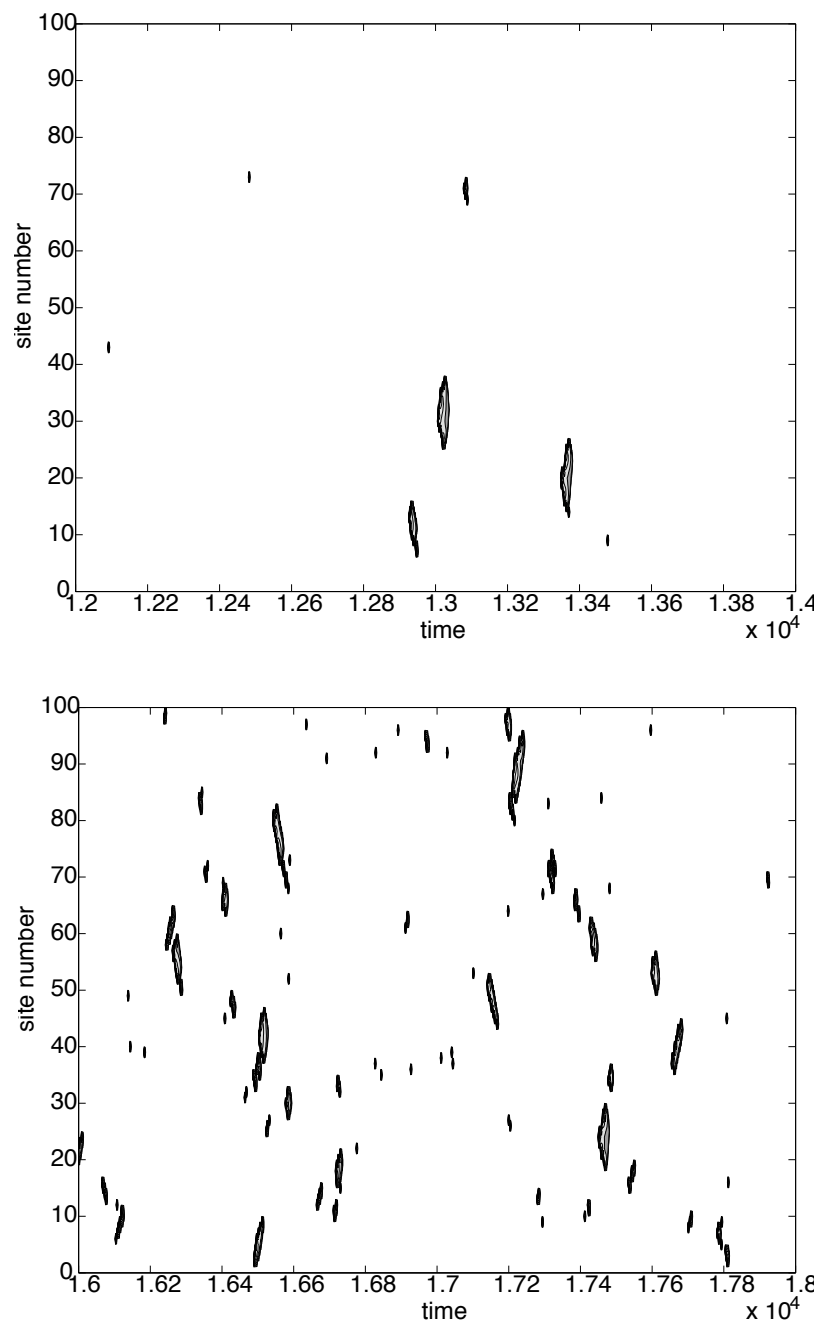

Fig. 2. Closer look at Fig. 1.

The mean radiated energy would be $(1-a) J_{a v}$. Respectively, $n_{a v}=\sum_{i, t} \theta(J) /\left(L N_{t}\right)$ would give the mean fractional number of active sites. We remind the reader that the mean energy input per site is $q p$. In the stationary regime $(1-a) J_{a v} \approx q p$ (the equality cannot be precise because of the losses at the boundaries - see complete analysis in section 4). The results of this comparison are given in Table 1, where we list the following parameters: $p$ is the driving probability, $q p$ is the average driving input per site, $J_{a v}$ is the average power released by active sites, $n_{a v}$ is the mean fractional number of active
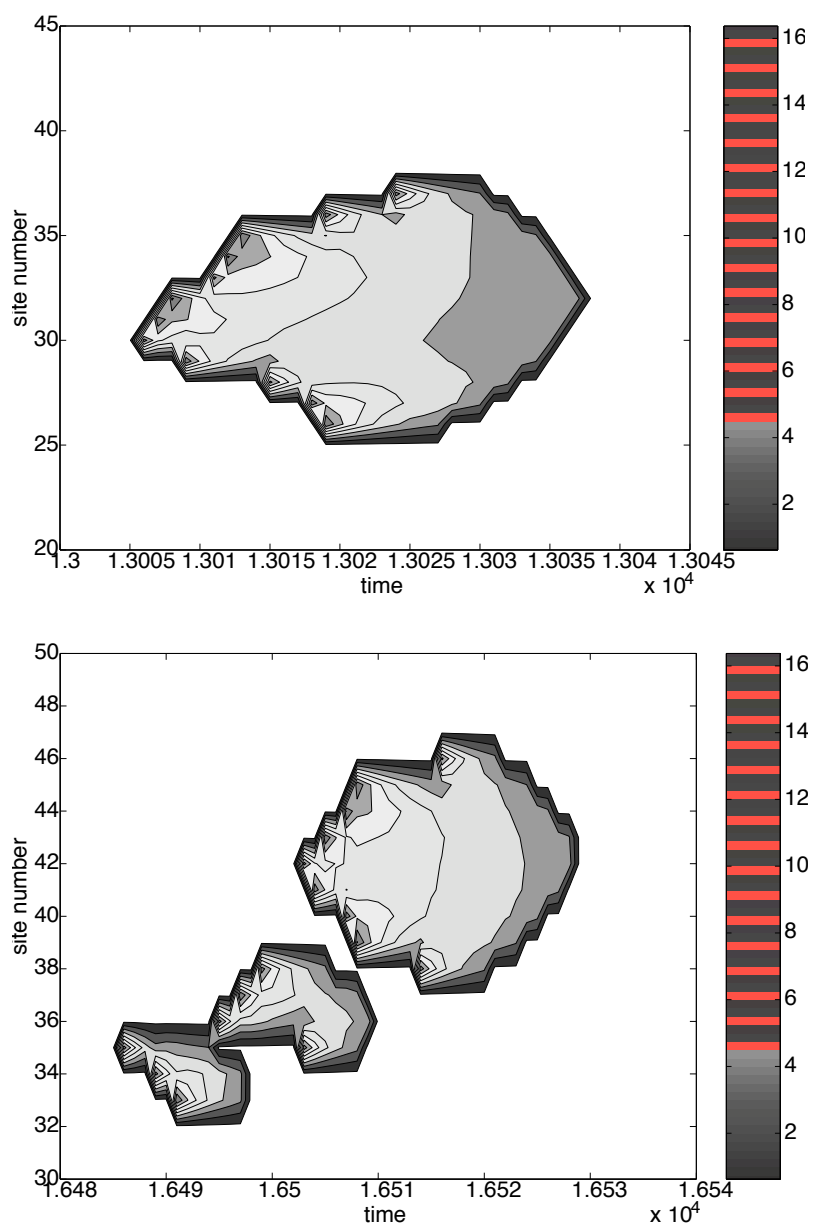

Fig. 3. Individual avalanche structure: top $p=0.0005$ and bottom $p=0.005$.

sites, $w_{\max }$ is the largest cluster size (from all cluster sizes measured at all times), and $t_{\max }$ is the longest avalanche duration (measured for all sites). The last two are defined as follows. First we determine the largest cluster size $\max (w(t))$ for each time $t$ and then $w_{\max }=\max _{t}(\max (w(t)))$. The longest duration $\max (t(i))$, on the other hand, is determined for each site separately, and then $t_{\max }=\max _{i}(\max (t(i)))$. Thus, $w_{\max }$ corresponds to the time-average spatial pattern which an observer would see instantaneously, while $t_{\max }$ corresponds to the spatially-average time evolution which an observer, sitting at some site, would see.

Figure 2 provides a close look at the avalanche-cluster distribution for different drivings. It is seen that, besides much more frequent bursts of activity, in a number of cases the avalanches for stronger driving behave in a more complicated way. This can be seen from Fig. 3 where individual avalanches are shown. The stronger driving case exhibits first indications of avalanche merging. At stronger drivings such merging would become more important, thus spoiling our analytical treatment.

For the analysis of the distribution $N(w)$ the size of the system was increased to $L=400$, in order to exclude the 


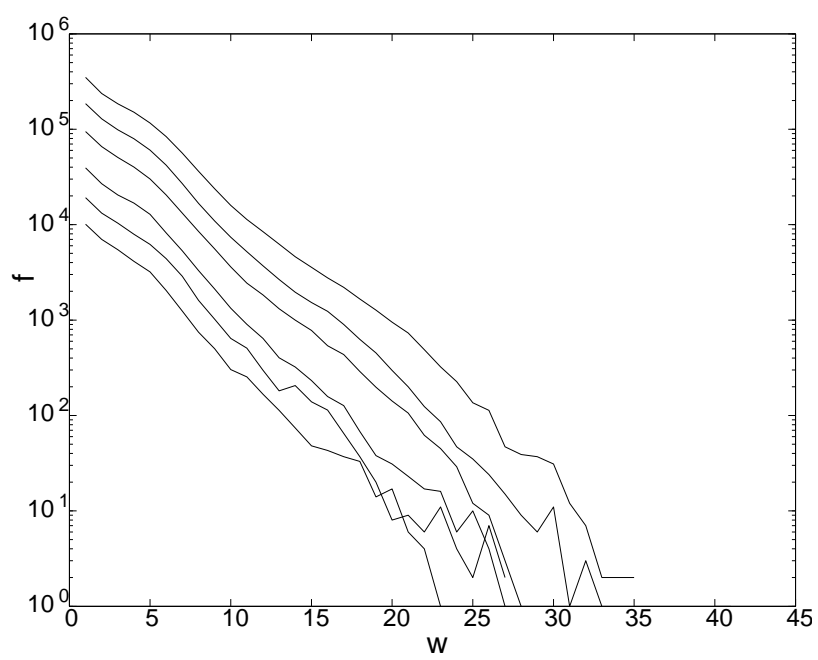

Fig. 4. Distribution $N(w)$ (log-linear scale) for $p=0.00025,0.0005,0.001,0.0025,0.005$.

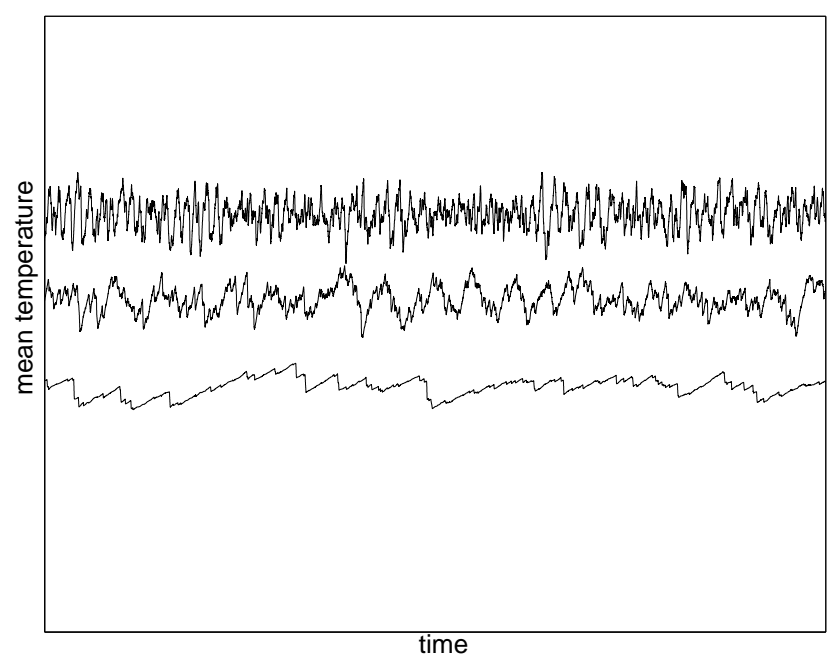

Fig. 5. Mean temperature for various drivings (shifted).

effects of the edges. Figure 4 shows the distribution for various values of the probability $p$. As expected, the maximum cluster size depends only weakly on the driving, and the functional dependence $\ln N \propto-w$ remains the same.

Figure 5 shows the behavior of the mean temperature. The plots are artificially shifted since the dependence of the temperature on the driving is negligible. It is seen that the system is in the stationary regime, since the temperature fluctuates around some constant value. With increase of driving the fluctuations become more frequent.

We conclude the illustration of the system behavior with plots of the duration of active and passive phases, which are distinguished by $J_{t}=\sum_{i} J_{i}$. An active phase corresponds to $J_{t}>0$, that is, there is at least one active site. In the passive phase there is no activity at all. The statistics is given in Fig. 6.
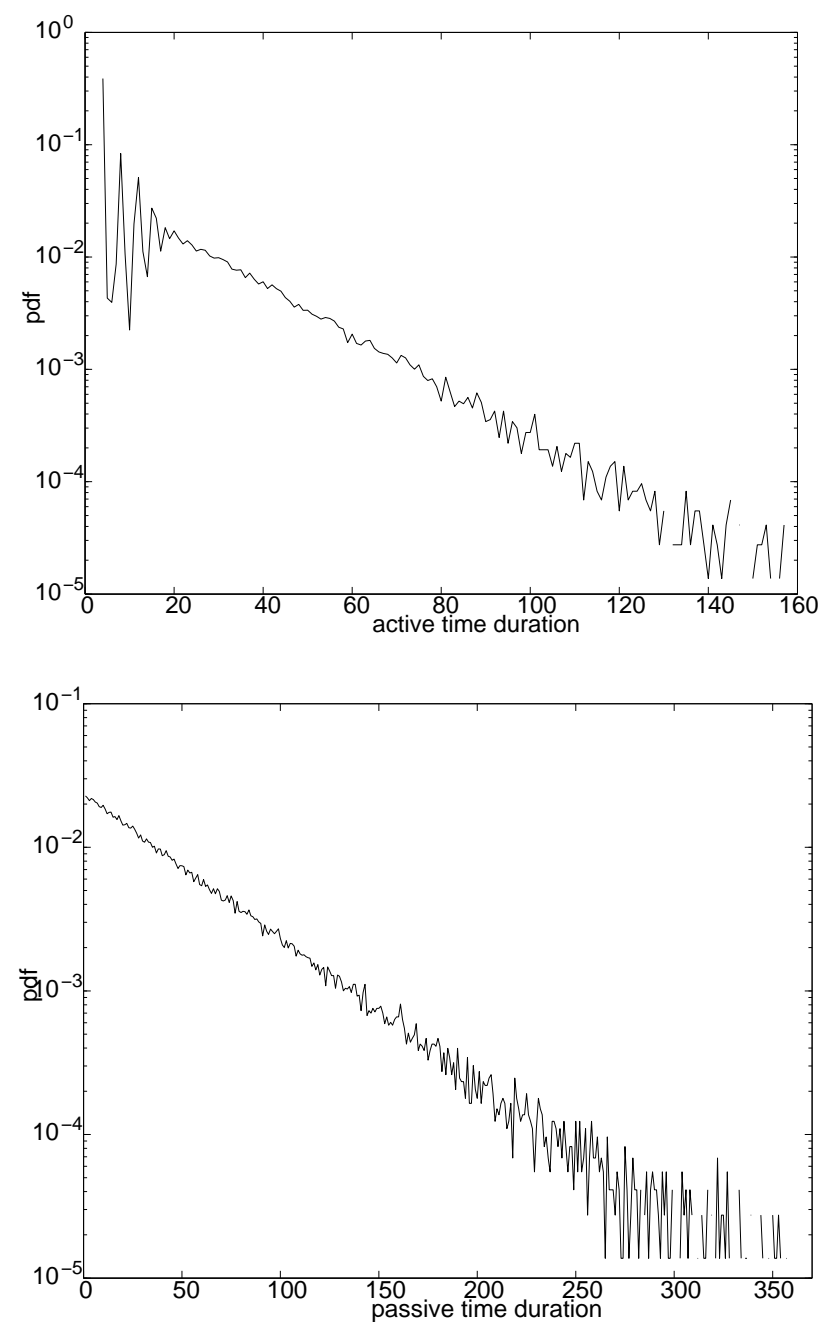

Fig. 6. Duration (pdf) of the active (top) and passive (bottom) phases.

The distribution of the passive phase durations is Poisson in a wide range, as could be expected. The Poisson nature of the PDF of passive phases suggests that the evolution of the burning model as described in this manuscript does not show any time correlation among the avalanche events. However, further work is needed in order to investigate the emergence of time correlation as a function of the driving strength and/or different updating rules including diffusion effects. The distribution of the active phase durations deviates from Poisson toward smaller and larger durations. More detailed comparative analysis of the statistical behavior of avalanches is provided in the companion paper.

\section{Conclusions}

In the present paper we proposed a new model which seems to be more appropriate for phenomenological description of magnetized plasma systems with localized reconnection (such as solar flares and reconnection in Earth's magnetotail), 
than the usually used sandpile models. The features of the model allow to formulate it in continuous form (as field equations) and as a discrete mapping model as well. Rather simple and precise analytical treatment appears to be possible, so that we can predict a certain shape of the cluster distribution for the stationary regime. Numerical analysis supports the results of the analytical study. The statistical properties of this model are studied in the companion paper, in comparison with a similar sandpile model. The two-dimensional generalization of the model will be published elsewhere.

Acknowledgements. This work has been performed within the framework of the "Observable features of avalanching systems" ISSI Science Team.

Edited by: N. Watkins

Reviewed by: Z. Voros, A. Klimas, and H. Jeldtoft Jensen

\section{References}

Bak, P., Tang, C., and Wiesenfeld, K.: Self-organized criticality An explanation of $1 / f$ noise, Phys. Rev. Lett., 59, 381-384, 1987.

Bak, P., Tang, C., and Wiesenfeld, K.: Self-organized criticality, Phys. Rev. A, 38, 364-374, 1988.

Bak, P. and Tang, C.: Earthquakes as a self-organized critical phenomena, J. Geophys. Res., 94, 15 635-15 637, 1989.

de Boer, J., Derrida, B., Flyvbjerg, H., Jackson, A. D., and Wettig, T.: Simple model of self-organized biological evolution, Phys. Rev. Lett., 73, 906-909, 1994.

Boffetta, G., Carbone, V., Giuliani, P., Veltri, P., and Vulpiani, A.: Power laws in solar flares: Self-organized criticality or turbulence? Phys. Rev. Lett., 83, 4662-4665, 1999.

Chang, T.: Self-organized criticality, multi-fractal spectra, sporadic localized reconnections and intermittent turbulence in the magnetotail, Phys. Plasmas, 6, 4137-4145, 1999.

Chapman, S. C., Watkins, N. W., Dendy, R. O., Helander, P., and Rowlands, G.: A simple avalanche model as an analogue of for magnetospheric activity, Geophys. Res. Lett., 25, 2397-2400, 1998.

Charbonneau, P., McIntosh, S. W., Liu, H.-L., and Bogdan, T. J.: Avalanche models for solar flares (Invited Review), Solar Physics, 203, 321-353, 2001.

Consolini, G. De Michelis, P.: A revised forest-fire cellular automaton for the nonlinear dynamics of the Earth's magnetotail, JASTP, 63, 1371-1377, 2001

Corral, A. and Paczuski, M.: Avalanche merging and continuous flow in a sandpile model, Phys. Rev. Lett., 83, 572-575, 1999.

Hamon, D., Nicodemi, M., and Jensen, H. J.: Continuously Driven OFC: A Simple Model of Solar Flare Statistics, Astron. Astrophys., 387, 326-334, 2002.

Hwa, T. and Kardar, M.: Avalanches, hydrodynamics, and discharge events in models of sandpiles, Phys. Rev. A, 45, 70027027, 1992.
Jensen, H. J.: Self-Organized Criticality: emergent Complex Behavior in Physical and Biological Systems, Cambridge University Press, 1998.

Klimas, A. J., Uritsky, V. M., Vassiliadis, D., and Baker, D. N.: Reconnection and scale-free avalanching in a driven current-sheet model, J. Geophys. Res., 109, 2218-2231, 2004.

Krasnoselskikh, V., Podladchikova, O., Lefebvre, B., and Vilmer, N.: Quiet Sun coronal heating: a statistical model, Astron. Astrophys., 382, 699-712, 2002.

Lu, E.T.: Avalanches in continuum driven dissipative systems, Phys. Rev. Lett., 74, 2511-2514, 1995.

Lu, E., and Hamilton, R. J.: Avalanches and the distribution of solar flares, Astrophys. J., 380, L89-92, 1991.

Lu, Y. N. and Ding, E. J.: Self-organized criticality in elastic springblock model, Phys. Rev. E, 48, 21-24, 1993.

Milovanov, A. V., Zelenyi, L. M., Zimbardo, G., and Veltri, P.: Selforganized branching of magnetotail current systems near the percolation threshold, J. Geophys. Res., 106, 6291-6307, 2001.

Milovanov, A. V. and Zelenyi, L. M.: Nonequilibrium stationary states in the earth's magnetotail: stochastic acceleration processes and nonthermal distribution functions, Advances in Space Research, 30, 2667-2673, 2002.

Sanchez, R., Newman, D. E., and Carreras, B. A.: Waiting-Time Statistics of Self-Organized-Criticality Systems, Phys. Rev. Lett., 88, 8302-8305, 2002.

Semenov, V.S., Kubyshkin, I.V., Lebedeva, V.V., Rijnbeek, R.P., Heyn, M.F., Biernat, H.K., and Farrugia, C.J.,: A comparison and review of steady-state and time varying reconnection, Planet. Space Sci., 40, 63-87, 1992.

Sergeev, V.A., Semenov, V.S., and Sidneva, M.V.: Impulsive reconnection in the magnetotail during substorm expansion, Planet. Space Sci., 35, 1199-1212, 1987.

Slavin, J.A., Lepping, R.P., Gjerloev, J., Fairfield, D.H., Hesse, M., Owen, C.J., Moldwin, M.B., Nagai, T., Ieda, A., and Mukai, T., Geotail observations of magnetic flux ropes in the plasma sheet, J. Geophys. Res., 108, 1015-1032, 2003.

Takalo, J., Timonen, J., Klimas, A. J., Valdivia, J. A., and Vassiliadis, D.: A coupled-map model for the magnetotail current sheet, Geophys. Res. Lett., 26, 2913-2916, 1999.

Valdivia, J.A., Klimas, A., Vassiliadis, D., Uritsky, V., and Takalo, J.: Self-organization in a current sheet model, Sp. Sci. Rev., 107, 515-522, 2003.

Uritsky, V., Pudovkin, M., and Steen, A.: Geomagnetic substorms as perturbed self-organized critical dynamics of the magnetosphere, Journal of Atmospheric and Terrestrial Physics, 63 , 1415-1424, 2001.

Uritsky, V. M., Klimas, A. J., and Vassiliadis, D.: Multiscale dynamics and robust critical scaling in a continuum current sheet model, Physical Review E, 65, 6113-6117, 2002.

Zelenyi, L. M., Delcourt, D. C., Malova, H. V., Sharma, A. S., Popov, V. Y., and Bykov, A. A.: Forced current sheets in the Earth's magnetotail: their role and evolution due to nonadiabatic particle scattering, Advances in Space Research, 30, 1629-1638, 2002. 